\title{
Application of Fuzzy Support Vector Machine for Determining the Health Index of the Insulation System of In- service Power Transformers
}

\author{
Atefeh Dehghani Ashkezari, Hui Ma, Tapan K. Saha, and Chandima Ekanayake \\ The University of Queensland, \\ Brisbane, Australia
}

\begin{abstract}
With the integration of data and information obtained from a variety of chemical and electrical tests on transformer insulating oil, it is possible to evaluate the health condition of the insulation system of an in-service power transformer. This paper develops an intelligent algorithm for automatically processing the data collected from oil tests and determining a health index for the transformer insulation system. This intelligent algorithm adopts a fuzzy support vector machine (FSVM) approach, which constructs a statistical model using a training database based on the historic data collected from 181 in-service power transformers. The procedure of constructing the training database, the formulation and implementation of FSVM and the data preprocessing methods for dealing with a class imbalanced training database is presented in this paper. Numerical experiments are also conducted to evaluate the performance of the algorithms developed in the paper.
\end{abstract}

Index Terms - Condition assessment of transformer, health index, insulation system, oil test, power transformer, fuzzy support vector machine (FSVM), support vector machine (SVM).

\section{INTRODUCTION}

POWER transformer is one of the most critical assets in the electricity supply chain [1]. It is therefore necessary to develop reliable and effective techniques to provide a comprehensive assessment on transformer condition to facilitate the decision making involving transformer operation and maintenance [2-4]. Recently, a health index (HI) method has been proposed for power transformer condition assessment. In this approach, it firstly collects a variety of data and information from operating observations, field inspections, online and offline measurements and then performs scoring and weighting on different measurements and tests results on the basis of utilities practices and related standards (i.e. IEC, IEEE and CIGRE recommendations) [5]. Finally, it computes an index value regarding the health condition of the transformer under investigation.

However, the approaches reported in the literature for computing the health index of in-service transformers still suffer some limitations. For example, the calculated health indexes might not be consistent. This is because the scoring and weighting in the health index computation are subjective in nature (i.e. decided by utility experts) and may vary from utility to utility. Moreover, on some occasions it might be difficult to provide an explicit health index on some combinations of data collected from different online and offline measurements. To address these limitations, this paper develops a suite of intelligent algorithms, which utilises the historic measurement data of oil tests and the insulation condition of corresponding transformer to construct a mathematical model to approximate the underlying relationship between oil tests data and transformer insulation condition.

This paper adopts a fuzzy support vector machine (FSVM) technique for automatically determining the health index of the insulation system of in-service transformers by using transformer oil characteristic test data. Two key issues of applying FSVM to the transformer health index computation are addressed in the paper. The first issue is the construction of a training database which will be utilised by the FSVM algorithm to exploit the underlying relationship between the oil test results and the health index of the transformer insulation system. The second issue is dealing with the imbalanced training dataset, in which the number of power transformers with poor insulation condition is limited. Such an imbalanced training dataset exhibits an unequal distribution between different levels of health index and normally have reverse impacts on the performance of FSVM.

The organization of this paper is as follows. Section 2 provides a brief introduction of different characteristic tests of 
transformer insulating oil. Section 3 describes the procedure of constructing the training dataset for FSVM. Section 4 presents the mathematical formulation and implementation of FSVM technique for determining the health index of the transformer oil-paper insulation system. A number of approaches for dealing with the effects of class imbalance in the training database are also presented in this section. Section 5 provides a case study to demonstrate the applicability of the developed algorithms in evaluating a transformer insulation system using the health index. Section 6 concludes the paper.

\section{BRIEF REVIEW OF TRANSFORMER OIL CHARACTERISTIC TESTS}

Mineral insulating oil is commonly used in power transformers. Ageing and various chemical and electrical stresses lead to the separation of molecular bonds of insulating oil and may generate fragments that can easily interact with each other or some external molecules. Consequently, various dissolved gases, moisture, acid components and some other contaminants might be formed inside the insulating oil [2]. Therefore, routine transformer oil characteristic tests are conducted by utilities to reveal the condition of transformer oil and the overall insulation system for ensuring the proper operation of transformers.

Among oil characteristic tests, Dissolved Gas Analysis (DGA) technique is one of the well-established preventative maintenance tools and its interpretation schemes include Doernenburg, Rogers, IEC Ratio, Duval's Triangle and Single Gas Ratio method [6]. DGA technique can identify transformer fault conditions such as internal arcing, partial discharge, overheating and hot spots [6]. Other than DGA, there are also a number of physical and chemical oil tests to reveal insulation conditions such as moisture test, acidity test, dielectric strength test, dielectric dissipation factor test and resistivity test [7]. In a transformer the moisture content comes from the atmosphere or it is produced due to the deterioration of solid insulating material, e.g. paper/pressboard [7]. When moisture content increases beyond the oil saturation level, free water will be formed and as a result the electrical strength and resistivity of insulating oil will decrease [8]. Acids mainly originate from oxidation products in the insulating oil [9]. They can also be produced from atmospheric contamination [9]. Together with moisture content and solid contaminants, acids and other oxidation products will affect different properties of insulating oil [8]. The dielectric losses in the oil can be measured by dielectric dissipation factor (DDF) test. Results obtained from this test are very sensitive to the presence of ageing products and soluble polar contaminants in the insulating oil [8]. Hence, the DDF measurement result is a good indication of the contaminant variations in insulating oil. Moreover, oil resistivity measures the degree of losses in the insulating oil and breakdown voltage (BDV) of insulating oil measures the strength of oil to withstand electrical stress without any failure [10], [11]. In principle, the condition of transformer insulating oil can be evaluated using the above oil characteristic tests [12].
On the other hand, various thermal and electrical stresses may also eventually cause the decomposition of cellulose paper and lead to the formation of water and carbon oxides $\left(\mathrm{CO}\right.$ and $\mathrm{CO}_{2}$ ) [2]. Moreover, the paper ageing by-product can be detected through the measurement of 2-furfuraldehyde in insulating oil. Therefore, among the above oil characteristic tests, 2-furfuraldehyde test and measurement of dissolved carbon oxides in oil can be used to evaluate the condition of paper insulation in a transformer. This paper makes use of the oil characteristic test results to evaluate the condition of transformer oil-paper insulation and subsequently determine the health index for a transformer insulation system.

\section{COMPREHENSIVE METHOD OF CONSTRUCTING TRAINING DATABASE}

This paper applies fuzzy support vector machine (FSVM) to compute the health index of transformer insulation systems. In FSVM, a training database consisting of oil characteristic test results with already known transformer insulation conditions is required. By using such a database, it is possible for FSVM to find optimal parameters and build up a mathematical model to predict a health index for a transformer of interest (this transformer is not in the training database).

This section details the procedure of constructing a training database, in which each record consists of the oil characteristic test results of an in-service transformer and its health index. In this paper, the health index is categorized into five different levels by combining the diagnosis results obtained from industry standards, Duval's triangle method and fuzzy $c$-means algorithm. Moreover, utility experts' judgments are also incorporated into the health index calculation as a supplement to the above three methods.

\subsection{DETERMINING HEALTH INDEX ON THE BASIS OF INDUSTRY STANDARDS $\left(\mathrm{HI}_{1}\right)$}

This section computes the health index by using a similar approach as proposed in [5], which is based on ASTP, IEC and IEEE standards and CIGRE recommendations. By considering different dissolved gas limits recommended by the standards [13, 14], the DGA Factor (DGAF) on determining the health index can be defined as:

$$
D G A F=\frac{\sum_{i=1}^{7} S_{i} \times W_{i}}{\sum_{i=1}^{7} W_{i}}
$$

where $i$ denotes seven dissolved gases $\left(\mathrm{H}_{2}, \mathrm{CH}_{4}, \mathrm{C}_{2} \mathrm{H}_{6}, \mathrm{C}_{2} \mathrm{H}_{4}\right.$, $\mathrm{C}_{2} \mathrm{H}_{2}, \mathrm{CO}$ and $\mathrm{CO}_{2}$ ), $S_{i}$ is the score value based on the volume of dissolved gases, and $W_{i}$ represents the weighting factor of each individual dissolved gas [5].

Similarly, by considering the oil test limits recommended by IEEE, ASTM and IEC $[15,16]$, the Oil Quality Factor (OQF) on determining the health index can be defined as: 


$$
O Q F=\frac{\sum_{i=1}^{4} S_{i} \times W_{i}}{\sum_{i=1}^{4} W_{i}}
$$

where $i$ denotes four oil tests (BDV, acidity, moisture content and DDF), $S_{i}$ is the score based on the value of oil test results and $W_{i}$ represents the weighting factor of each individual oil test [5]. Moreover, the Paper Insulation Factor (PIF) on determining the health index is defined based on the amount of furfural content of the insulating oil, which can be traced using IEC 61198 [17]. Table 1 shows the PIF values based on their level of furfural content. In the case of the transformer oil having been changed, the information by 2 -furfuraldehyde test cannot determine the paper degradation; therefore, PIF receives a low weighting value in the final Health Index calculation using Equation 3.

Table 1. 2-furfuraldehyde test rating [5].

\begin{tabular}{|c|c|}
\hline Rating Code & $\begin{array}{c}\text { Concentration } \\
{[\mathrm{ppm}]}\end{array}$ \\
\hline 1 & $0-0.1$ \\
\hline 2 & $0.1-0.5$ \\
\hline 3 & $0.5-1$ \\
\hline 4 & $1-5$ \\
\hline 5 & $>5$ \\
\hline
\end{tabular}

Finally, the above three factors, i.e. DGAF, OQF, and PIF are combined into a single health index as defined by Equation 3:

$$
H I_{1}=\frac{\sum_{j=1}^{3} F_{i} \times K_{i}}{\sum_{j=1}^{3} K_{i}}
$$

where $F_{i}$ represents factor values of DGAF, OQF and PIF. $K_{i}$ values for DGAF, OQF and PIF are 10, 8 and 4 respectively which are decided based on the importance and reliability of diagnosis techniques included in each factor $\left(F_{i}\right)$.

The health index value obtained from Equation 3 is normalized into five levels from level 1 to level 5, which correspond to excellent, good, fair, poor and unsatisfactory conditions of a transformer insulation system.

\subsection{DETERMINING HEALTH INDEX ON THE BASIS OF UTILITY EXPERTS' JUDGEMENTS $\left(\mathrm{HI}_{2}\right)$}

Along with oil characteristic tests results, utility experts have access to further information regarding the transformer under investigation. The information may include loading history, operating conditions (temperature and weather conditions), maintenance history and the information obtained from a visual inspection. Moreover, the utility experts have well-established knowledge and expertise to analyse every piece of information to make an assessment on the condition of a transformer.

In this paper the oil test records provided by the utility are accompanied with utility expert comments on the condition of each transformer. In order to incorporate expert advice into the health index, this paper quantifies expert comments into five different levels of excellent, good, fair, poor and unsatisfactory regarding insulation conditions of a transformer.

\subsection{DETERMINING HEALTH INDEX ON THE BASIS OF DGA INTERPRETATION RESULTS $\left(\mathrm{HI}_{3}\right)$}

DGA is one of the most important tools for transformer fault diagnosis. Its interpretation methods include Dornenburg Ratios, Rogers Ratios, Duval's Triangle and IEC/IEEE standards. In the authors previous study, it has been found that the Duval's Triangle could attain a higher diagnosis accuracy compared to other methods [6]. In this paper, the DGA data of each oil test record was evaluated by Duval's Triangle method. According to the faults identified by Duval's Triangle method, the corresponding transformer insulation condition (health index) is quantified into five levels, from level one of excellent condition to level five of severe condition as shown in Table 2.

Table 2. Duval triangle rating.

\begin{tabular}{|c|c|}
\hline Rating Code $\left(\mathrm{HI}_{3}\right)$ & Fault detected by Duval Triangle method \\
\hline 1 & Normal \\
\hline 2 & Normal but high TCG \\
\hline 3 & D1, T1 \\
\hline 4 & PD, T2 \\
\hline 5 & D2,T3 \\
\hline \multicolumn{2}{|c|}{ Note: TCG: Total Combustible Gases, D1: discharge of low energy, D2: discharge of high } \\
energy, T1: low thermal fault, T2: medium thermal fault, T3: high thermal fault, PD: Partial
\end{tabular}

\subsection{DETERMINING HEALTH INDEX USING FUZZY C- MEANS ALGORITHM $\left(\mathrm{HI}_{4}\right)$}

Due to measurement errors and influences from any environmental interference, there may exist a certain degree of inaccuracy to oil tests data (i.e. noises). This may cause a discrepancy among the above three sets of health indices, which are determined by industry standards, expert judgements and Duval's Triangle respectively.

To deal with noisy data and to assure the consistency of the health index calculated by different methods, this paper adopts the Fuzzy $C$-Means (FCM) algorithm to investigate which extent that each oil test record belongs to one particular health index (i.e. health index from level one to level five).

FCM is an unsupervised clustering method, in which data can belong to more than one cluster (group) with different membership values. The membership value indicates the confidence of the association between that data and a particular cluster [18]. Fuzzy clustering is a process of assigning these membership levels and then using them to assign data elements to one or more clusters [18]. In this paper, FCM is used to calculate membership values for each oil test record with respect to each of the five clusters (each cluster corresponds to one health index level). Then FCM assigns the above test record to the cluster, to which this record has the highest membership value.

\subsection{ASSIGNING FINAL HEALTH INDEX AND FORMING THE TRAINING DATABASE (HI)}

After obtaining four sets of health index based on industry standard, Duval's Triangle method, Fuzzy $C$-means algorithm 
Table 3. Examples of determination of Health Index for transformer insulation system

\begin{tabular}{|c|c|c|c|c|c|c|c|c|c|c|c|c|c|}
\hline \multirow[t]{2}{*}{$\begin{array}{l}\text { Trans. } \\
\text { Num. }\end{array}$} & \multicolumn{4}{|c|}{$\begin{array}{l}\text { Determining Health Index Based } \\
\text { on Industry Standards }\end{array}$} & \multirow{2}{*}{$\begin{array}{l}\text { Expert Judgement } \\
\qquad \mathrm{HI}_{2}\end{array}$} & \multirow{2}{*}{$\begin{array}{c}\text { Duval's Triangle } \\
\mathrm{HI}_{3}\end{array}$} & \multicolumn{6}{|c|}{$\mathrm{FCM}$} & \multirow{2}{*}{$\begin{array}{c}\text { Final } \\
\mathrm{HI}^{*}\end{array}$} \\
\hline & DGAF & OQF & PIF & $\mathrm{HI}$ & & & M1 & M2 & M3 & M4 & M5 & $\mathrm{HI}$ & \\
\hline 1 & 1 & 1 & 1 & 1 & 1 & 1 & 0.94 & 0.06 & 0.00 & 0.00 & 0.00 & 1 & 1 \\
\hline 2 & 1 & 1 & 1 & 1 & 1 & 1 & 0.93 & 0.06 & 0.01 & 0.00 & 0.00 & 1 & 1 \\
\hline 3 & 1 & 2 & 2 & 2 & 1 & 2 & 0.43 & 0.54 & 0.02 & 0.01 & 0.00 & 2 & 2 \\
\hline 4 & 2 & 1 & 2 & 2 & 2 & 2 & 0.27 & 0.70 & 0.02 & 0.01 & 0.00 & 2 & 2 \\
\hline 5 & 5 & 1 & 1 & 3 & 3 & 3 (D1) & 0.10 & 0.10 & 0.77 & 0.04 & 0.00 & 3 & 3 \\
\hline 6 & 4 & 1 & 2 & 3 & 3 & 2 & 0.14 & 0.14 & 0.66 & 0.07 & 0.00 & 3 & 3 \\
\hline 7 & 5 & 1 & 4 & 4 & 4 & $4(\mathrm{~T} 2)$ & 0.24 & 0.26 & 0.23 & 0.25 & 0.01 & 2 & 4 \\
\hline 8 & 5 & 1 & 4 & 4 & 4 & 4 (PD) & 0.15 & 0.15 & 0.16 & 0.27 & 0.26 & 4 & 4 \\
\hline 9 & 5 & 5 & 3 & 5 & 5 & 5 (D2) & 0.16 & 0.17 & 0.57 & 0.10 & 0.01 & 3 & 5 \\
\hline 10 & 5 & 4 & 4 & 5 & 5 & 5 (T3) & 0.19 & 0.19 & 0.20 & 0.21 & 0.20 & 4 & 5 \\
\hline
\end{tabular}

and expert judgement, the task is to combine these four sets of health index into one final health index. Since the four methods compute the health index based on different criteria, there may exist discrepancies among different methods. To deal with such possible discrepancies, the majority vote strategy is adopted for deciding the final health index level. Table 3 exemplifies the determination of final health index for the insulation system of ten in-service transformers.

It can be seen from Table 3 that the health index ranked by four assessment criteria are consistent for health index level one (excellent condition) and level two (good condition). For example, FCM assigns the related records (data) into these two health index levels with a high membership value (94\%, and 93\% in Level one, 54\% and 70\% in Level Two) and Duval's Triangle identifies the transformers are in normal condition. Thus the determinations of final health index for health index level one and two are straightforward. It can also be seen that for health index level three, four assessment criteria only have a slight discrepancy.

Correctly determining health index levels four and five for transformer insulation system is crucial since these two health index levels indicate the faults are occurring in a transformer insulation system and immediate action needs to be taken. As shown in Table 3 , transformers 7 to 10 are assigned with a health index of level four or five based on both industry standards criteria and utilities expert judgements. Duval's Triangle also reveals these four transformers may have either medium to high temperature overheat or partial discharge or discharge of high energy faults. However, FCM could not make explicit determination of the health index level for these four transformers. Based on the majority vote, transformers 7 and 8 are assigned with health index level four since three out of four criteria indicate the health index level is at four. Similarly, transformers 9 and 10 are assigned with health index level five since three out of four criteria indicate the health index level is five.

The above procedure of determining the health index level for a transformer insulation system is applied to 181 power transformers to form the training database. Table 4 presents the distribution of health index levels of this database. This database will be used for training the FSVM algorithm, which can then automatically determine the health index.
Table 4. The Assigned Health Index of 181 Oil Power transformers

\begin{tabular}{|c|c|c|c|}
\hline $\begin{array}{c}\text { Health Index } \\
\text { Levels }\end{array}$ & $\begin{array}{c}\text { Insulation System } \\
\text { Condition }\end{array}$ & $\begin{array}{c}\text { Transformer } \\
\text { Number in } \\
\text { Corresponding } \\
\text { Health Index Levels }\end{array}$ & $\begin{array}{c}\text { Percentage } \\
(\%)\end{array}$ \\
\hline 1 & Excellent & 80 & 44 \\
\hline 2 & Good & 50 & 27 \\
\hline 3 & Fair & 21 & 12 \\
\hline 4 & Poor & 20 & 11 \\
\hline 5 & Unsatisfactory & 10 & 6 \\
\hline Total & & 181 & 100 \\
\hline
\end{tabular}

\section{FUZZY SUPPORT VECTOR MACHINE (FSVM) FOR AUTOMATICALLY DETERMINING HEALTH INDEX FOR TRANSFORMER OIL PAPER INSULATION}

This section presents the FSVM approach for automatically determining the health index for a transformer insulation system. This section starts with the introduction of support vector machine (SVM) and then presents the key ideas behind FSVM. Since the training database as shown in Table 4 is imbalanced (e.g. the transformers with health index level five are outnumbered by transformers with other health index levels) a number of pre-processing approaches are also implemented in this section.

\subsection{SUPPORT VECTOR MACHINE (SVM)}

On the basis of a statistical learning principle, the SVM is one of the commonly adopted machine learning techniques for data classification $[19,20]$. The SVM makes use of a training database, which is a collection of samples and each sample consists of a number of features. In this paper, each feature corresponds to a particular oil test result. Moreover, each sample in the above training database has been labelled with a target value (i.e. health index level), which is decided by industry standard, utility expert judgment, Duval's Triangle and Fuzzy c-means algorithm (refer to Section 3). Using this training database SVM will build up a mathematical model that can later be applied to determine the health index for the insulating system of an in-service transformer solely based on its oil characteristic test results. In general, the samples in the 
training database can be represented as $\mathbf{X}=\left[\mathbf{x}_{1}, \ldots, \mathbf{x}_{l}\right]$ and each sample belongs to one of the $K$ independent categories (health index levels) $\left[y_{1}, \ldots, y_{K}\right]$. Making use of the training dataset, the SVM is tasked to construct a hyperplane (model) to separate the samples into different categories. Such hyperplane (model) will later be used to classify any new sample into one of the above categories (health index levels).

To find the optimal separation hyperplane that maximizes the margin between different categories of samples in SVM algorithm, the samples are mapped into a higher dimensional feature space. The margin maximization is then formulated as a quadratic programming (QP) problem [19]:

$$
\begin{gathered}
\operatorname{Min}_{w, b, \xi} \frac{1}{2} \mathbf{w}^{T} \mathbf{w}+C \sum_{i=1}^{l} \xi_{i} \\
\text { s.t. } y_{i}\left(\mathbf{w}^{T} \phi\left(\mathbf{x}_{i}\right)+b\right) \geq 1-\xi_{i}, \quad \xi_{i} \geq 0 . \quad i=1, \ldots, l
\end{gathered}
$$

In Equation 4 , function $\emptyset$ maps the training samples $\mathbf{x}_{\mathrm{i}}$ into a higher dimensional space. The separation hyperplane is defined by $(\mathbf{w}, b)$. The slack variable $\xi_{\mathrm{i}}>0$ is an indication of misclassification error. Parameter $C$ in Equation 4 controls the trade-off between maximizing the margin and minimizing the number of misclassifications. The above QP problem can be transformed into its dual form with the introduction of the Lagrange multipliers $\alpha_{i}$ [19].

$$
\begin{gathered}
\operatorname{Max} W(\alpha)=\sum_{i=1}^{l} \alpha_{i}-\frac{1}{2} \sum_{i=1}^{l} \sum_{j=1}^{l} \alpha_{i} \alpha_{j} y_{i} y_{j} \phi\left(\mathbf{x}_{i}\right) . \phi\left(\mathbf{x}_{\mathrm{j}}\right) \\
\text { s.t. } \quad \sum_{i=1}^{l} y_{i} \alpha_{i}=0, \quad 0 \leq \alpha_{i} \leq C, \quad i=1, \ldots, l
\end{gathered}
$$

After obtaining the maximized hyperplane, SVM can predict the class label for new sample $\mathrm{x}$ as follows:

$$
y=\operatorname{sign}\left(\sum_{i=1}^{l} \alpha_{i} y_{i} \phi\left(\mathbf{x}_{i}\right) \phi(\mathbf{x})+b\right)
$$

\subsection{FUZZY SUPPORT VECTOR MACHINE (FSVM)}

In the above SVM algorithm all samples in the training dataset are treated uniformly and thus are sensible to noises and outliers. To deal with noises and outliers in the training dataset, a fuzzy support vector machine (FSVM) is applied in this paper. In FSVM the samples are assigned with different weights; the sample closer to the centre of its belonging class is assigned with a higher weight [21]. FSVM is formulated as follows [22]:

$$
\begin{gathered}
\operatorname{Min}_{w, b, \xi} \frac{1}{2} \mathbf{w}^{T} \mathbf{w}+C \sum_{i=1}^{l} \rho_{i} \xi_{i} \\
\text { s.t. } \quad y_{i}\left(\mathbf{w}^{T} \phi\left(\mathbf{x}_{i}\right)+b\right) \geq 1-\xi_{i}, \quad \xi_{i} \geq 0 .
\end{gathered}
$$

where the original training dataset becomes $\left\{\mathbf{x}_{i}, y_{i}, \rho_{i}\right\}$, $i=1, \ldots, l$, and $\rho_{i}\left(0<\rho_{i}<1\right)$ are the weights. The dual form equation for FSVM with the Lagrange multipliers $\alpha_{i}$ can be calculated as [22]:

$$
\begin{gathered}
\operatorname{Max} W(\alpha)=\sum_{i=1}^{l} \alpha_{i}-\frac{1}{2} \sum_{i=1}^{l} \sum_{j=1}^{l} \alpha_{i} \alpha_{j} y_{i} y_{j} \phi\left(\mathbf{x}_{i}\right) . \phi\left(\mathbf{x}_{\mathrm{j}}\right) \\
\text { s.t. } \quad \sum_{i=1}^{l} y_{i} \alpha_{i}=0, \quad 0 \leq \alpha_{i} \leq \rho_{i} C, \quad i=1, \ldots, l
\end{gathered}
$$

In order to calculate the weight for each sample in the training dataset, the geometric centre of each class needs to be decided. This can be achieved by calculating the Euclidean mean of the samples of each class. After obtaining the centres of each class in the training dataset, the sample weight $\rho\left(\mathbf{x}_{i}\right)$ can be calculated by using an exponentially decaying function [22]:

$$
\rho\left(\mathbf{x}_{i}\right)=\frac{2}{1+\exp \left(\beta d_{i}^{c e n}\right)}, \quad \beta \in[0,1]
$$

where $d_{i}^{c e n}=\left\|\mathbf{x}_{i}-\mathbf{c}_{i}\right\|^{1 / 2}$ is the Euclidean distance between the sample $\mathbf{x}_{i}$ and its cluster center $\mathbf{c}_{i}$. In this equation $\beta$ determines the steepness of the decay.

\subsection{PRE-PROCESSING METHODS FOR DEALING WITH CLASS IMBALANCE}

The training database (Table 4) exhibits an unequal distribution between different classes (health index levels); the samples in health index level five are significantly outnumbered by the samples in other health index levels. Such phenomenon is called a class imbalance problem and may lead to biased classification. This paper implements three data preprocessing methods namely over-sampling, under-sampling and Synthetic Minority Over-Sampling technique (SMOTE) to deal with class imbalance problems in the training database. These three methods will be integrated with SVM and FSVM for determining the health index.

\subsubsection{OVER-SAMPLING}

Over-sampling changes the samples distribution in the training dataset by duplicating the samples with the higher cost of misclassification. The duplication process is a random over-sampling with replacement and it will be repeated until the numbers of different training samples are proportional to their costs of misclassification. For a classification problem with $K$ classes, the function Cost $[i, c](i=1, \ldots K, c=1, \ldots K)$ represents the cost of misclassifying a sample of the $i$-th class to the $c$-th class. The overall misclassification cost of each class can be calculated as follows [23]:

$$
\operatorname{Cost}[i]=\sum_{c=1}^{C} \operatorname{Cost}[i, c]
$$

After calculating the misclassification cost of each class, the number of training samples $N_{k}^{*}$ in the $k$-th class after resampling can be decided as [23]:

$$
N_{k}^{*}=\left[\frac{\operatorname{Cost}[k]}{\operatorname{Cos} t[\lambda]} N_{\lambda}\right]
$$

where $\lambda$ refers to the class which has the smallest number of training samples to be duplicated and it can be identified as [23]:

$$
\lambda=\arg \min _{j} \frac{\frac{\operatorname{Cost}[j]}{\min _{c} \operatorname{Cost}[c]} N_{\arg \min \operatorname{Cost}[c]}}{N_{j}}
$$

If $N_{k}^{*}>N_{k}\left(N_{k}\right.$ is the sample number of $k$-th class before oversampling), then $\left(N_{k}^{*}-N_{k}\right)$ number of training samples in 
the $k$-th class should be re-sampled [23].

\subsubsection{UNDER-SAMPLING}

Under-sampling modifies the training sample distribution by deleting some training samples with lower costs of misclassification. This process will continue until the sample number of different classes is proportional to their costs of misclassification. As in the over-sampling, the $k$-th class will have $N_{k}^{*}$ training samples after re-sampling. This can be computed according to Equation 11 . However, $\lambda$ refers to the class which has the smallest number of training samples to be removed [23]:

$$
\lambda=\arg \max _{j} \frac{\frac{\operatorname{Cos} t[j]}{\max _{c} \operatorname{Cost}[c]} N_{\text {arg } \max \operatorname{Cost}[c]}}{N_{j}}
$$

If $N^{*}{ }_{k}<N_{k}\left(N_{k}\right.$ is the number of $k$-th class before undersampling), then $\left(N_{k}-N_{k}^{*}\right)$ training samples in the $k$-th class should be removed [23].

\subsubsection{SYNTHETIC MINORITY OVER-SAMPLING TECHNIQUE (SMOTE) [24]}

SMOTE algorithm adopts a hybrid approach, which performs over-sampling on the minority classes and undersampling on the majority classes. It over-samples minority classes by keeping all the original samples and generating synthetic samples to join the minority classes. The procedure of generating synthetic samples for the minority class can be summarized as follows: (1) finding $k$-nearest neighbours for each sample in the minority class; (2) for an individual minority sample randomly selecting $j$ samples from its $k$ nearest neighbours based on the amount of total samples required; (3) generating the synthetic samples along the line segments connecting the minority sample and its $j$ neighbours; (4) adding the new samples to the dataset. The advantage of SMOTE's over-sampling over random over-sampling is that the decision region for the minority classes is larger and less specific. This helps to avoid the over fitting problem. On the other hand, in SMOTE the sample number of the majority classes will be reduced by the under-sampling method using Equations 10, 11 and 13.

\section{NUMERIC RESULTS AND ANALYSIS}

This section presents the numeric results of applying FSVM algorithm to determine the health index of a transformer insulation system. In this section, the dataset preparation, numeric simulation setup and the performance of a variety of algorithms is presented.

\subsection{TRAINING DATABASE ANALYSIS}

As mentioned earlier in this paper, the training database (Table 4) exhibits imbalanced class distributions; the samples in the health index level One and Two (excellent and good condition) outnumbers the samples in the health index levels three, four, and five (fair, poor and unsatisfactory conditions). Health index level five only has ten records. However, such a phenomenon is not unusual since the faulty rate of a transformer is rather low. As discussed in the previous section, this paper will adopt under-sampling, over-sampling and SMOTE approaches to deal with the class imbalanced problem.

On the other hand, due to the imprecise nature of measurement equipment and environmental noises, the insulating oil test data may have considerably high levels of outliers. Figure 1 illustrates the oil test and DGA test data distribution of the training database. It can be seen that some oil test data have considerably high outliers where the outliers are named based on their transformer ID. The outliers are far away from other data and may have reverse impacts on the classification performance of SVM. This paper applies FSVM algorithm, which assigns different weights to the samples. The outliers are far away from their class centres and will be assigned with lower weights in FSVM. It is expected that the introduction of sample weights will improve the classification accuracy. This will be proven in the next Section.

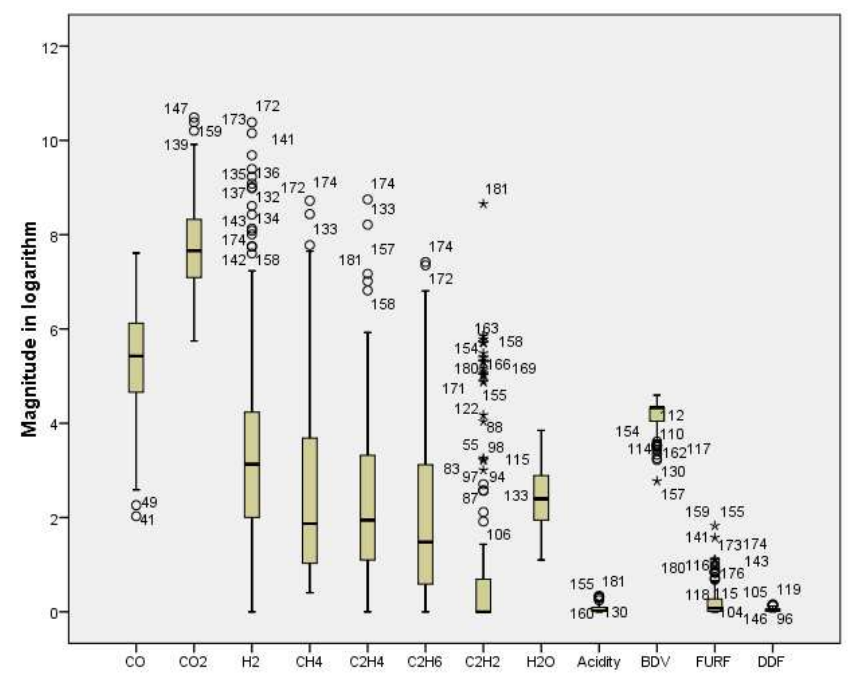

Figure 1. Distribution of transformer oil tests data in training database.

\subsection{NUMERIC EXPERIMENT SET-UP}

In the numeric experiment, SVM and FSVM algorithms and SVM and FSVM algorithms integrated with the three data preprocessing methods (under-sampling, over-sampling and SMOTE), a total of eight algorithms are implemented and evaluated. These eight algorithms are: SVM, SVM with undersampling, SVM with over-sampling, SVM with SMOTE, FSVM, FSVM with under-sampling, FSVM with oversampling and FSVM with SMOTE. In the algorithmic implementation, Libsvm software package has been used with the necessary modification and extension [25].

In the numeric experiment, the original database of 181 oil test records is randomly split into two parts of a training dataset and a testing dataset for validating the classification accuracy of the above eight algorithms, where $70 \%$ of samples of the original dataset considered for the training dataset and the remaining $30 \%$ for the testing dataset. 
The radial-basis function (RBF) kernel $K\left(\mathbf{x}_{i}, \mathbf{x}_{j}\right)=e^{-\gamma\left(\left\|\mathbf{x}_{i}-\mathbf{x}_{j}\right\|\right)^{2}}$ is adopted in eight algorithms. Tenfold cross validations are conducted on the training dataset to decide the optimal values for the pair of $(C, \gamma)$, where $C$ is the regularization parameter in SVM and FSVM to balance the margin maximization and misclassification and $\gamma$ is the variance parameter of RBF kernel [25]. The obtained optimal pair of $(C, \gamma)$ of each individual algorithm is then used to train the algorithm on the whole training dataset. Finally, the trained algorithms are applied to compute the health index level for the transformers in the testing dataset. It is worthy to point out that the data is scaled to $[-1,1]$ interval before running any algorithm. The above dataset split, cross-validation and testing will be repeated 10 times for each of the eight algorithms.

\subsection{NUMERIC EXPERIMENT RESULTS}

The numeric experiment results of SVM, SVM integrated with pre-processing methods, FSVM and FSVM integrated with pre-processing methods are summarized in Tables 5 and 6. Three different types of algorithm performance measures are investigated in this paper, which include the averaged classification accuracy on the testing dataset, the best classification accuracy on the testing dataset and the averaged classification accuracy for each class in the testing dataset.

It can be seen from Table 5 that SVM integrated with the different data pre-processing method attained different classification accuracy. SVM integrated with the undersampling method attained the lowest classification accuracy on the testing dataset compared to the original SVM algorithm (i.e. the SVM without the integration with any pre-processed methods). The under-sampling method is originally applied to the binary (two-class) classification problems [24]. However, this numeric experiment deals with a multiple class (five classes) dataset and has high sample population variations among different classes. This may lead to the undesirable classification accuracy of the SVM integrated with the undersampling method.

On the other hand, the SVM integrated with the oversampling method and SMOTE technique attained much better classification accuracy. As can be seen from Table 5, the average classification accuracy on the testing dataset of SVM integrated with over-sampling increased to $85 \%$ from $82 \%$ of the original SVM. The SVM integrated with SMOTE also significantly improves the classification accuracy on the testing dataset to about $86 \%$ from $82 \%$ of the original SVM.
Table 6 presents the classification accuracy obtained from FSVM algorithm with and without the integration of different data pre-processing methods. It can be seen that the FSVM integrating with the under sampling data pre-processing method has lower classification accuracy compared to the original FSVM (i.e. the FSVM without the integration with any pre-processing methods). Thus, it can be concluded that the under sampling method cannot effectively deal with the class imbalance problem and improve the classification accuracy of both SVM and FSVM algorithms in this numeric experiment. However, the FSVM integrated with oversampling and SMOTE methods attained higher classification accuracy compared to the original FSVM. The FSVM integrated with the SMOTE method attains the highest classification accuracy among seven algorithms in this case study, its overall averaged classification accuracy on the testing dataset is $87.8 \%$.

It can also be observed from Tables 5 and 6 that FSVM outperforms SVM. The averaged classification accuracy of the original SVM is $82.2 \%$ while that of the original FSVM is 84.1\%. FSVM integrated SMOTE also attains higher classification accuracy than SVM integrated with SMOTE does. The better performance of FSVM is due to its capability in dealing with outliers in the training dataset.

Tables 5 and 6 show that the classification accuracies of the samples for health index level five are lower than the classification accuracies for the rest of the health index levels. This is due to the small sample size of health index level five, which has only ten samples. As described in Table 4, the samples in health index level five correspond to the transformers with the critical conditions of their insulation system. And in the utilities the chance of having transformers with critical conditions of insulation system is relatively rare, thus only a few pieces of data are collected in this paper. However, SVM-SMOTE and FSVM-SMOTE still can improve the classification accuracy on the samples of health index level five to some extent. From the above discussions, it can be found that the SVM and FSVM integrated with oversampling and SMOTE could attain classification accuracy above $85 \%$. This demonstrates the applicability of the intelligent algorithms based on SVM and FSVM approaches for determining the health index of a transformer insulation system.

Table 5. Classification Result Obtained by SVM and SVM integrated with three pre-processing methods (averaged over 10 trials).

\begin{tabular}{|c|c|c|c|c|c|c|c|c|}
\hline \multirow[t]{2}{*}{ SVM } & \multirow[t]{2}{*}{ Algorithm } & \multirow{2}{*}{$\begin{array}{c}\text { Averaged classification } \\
\text { accuracy on testing dataset }\end{array}$} & \multirow{2}{*}{$\begin{array}{c}\text { Best classification accuracy } \\
\text { on testing dataset }\end{array}$} & \multicolumn{5}{|c|}{$\begin{array}{c}\text { Averaged classification accuracy of each class } \\
\text { on testing dataset }\end{array}$} \\
\hline & & & & 1 & 2 & 3 & 4 & 5 \\
\hline $\begin{array}{c}\text { Without Pre- } \\
\text { Processing Methods }\end{array}$ & SVM & 82.2 & 87.0 & 97.9 & 82.7 & 76.7 & 56.7 & 16.7 \\
\hline \multirow{3}{*}{$\begin{array}{l}\text { With Pre-Processing } \\
\text { Method }\end{array}$} & $\begin{array}{l}\text { SVM-Under } \\
\text { Sampling }\end{array}$ & 75.0 & 83.3 & 89.6 & 63.3 & 73.3 & 73.3 & 23.3 \\
\hline & $\begin{array}{l}\text { SVM- Over } \\
\text { Sampling }\end{array}$ & 85.6 & 94.4 & 98.3 & 88.7 & 75.0 & 68.3 & 23.3 \\
\hline & SVM-SMOTE & 85.9 & 92.6 & 98.8 & 76.7 & 81.7 & 80.0 & 50.0 \\
\hline
\end{tabular}


Table 6. Classification Result Obtained by FSVM and FSVM integrated with three pre-processing methods (average over 10 trials)

\begin{tabular}{|c|c|c|c|c|c|c|c|c|}
\hline \multirow{2}{*}{ FSVM } & \multirow{2}{*}{ Algorithm } & \multirow{2}{*}{$\begin{array}{l}\text { Average classification } \\
\text { accuracy on testing dataset }\end{array}$} & \multirow{2}{*}{$\begin{array}{c}\text { Best classification accuracy } \\
\text { on testing dataset }\end{array}$} & \multicolumn{5}{|c|}{$\begin{array}{c}\text { Average classification accuracy of each class on } \\
\text { testing dataset }\end{array}$} \\
\hline & & & & 1 & 2 & 3 & 4 & 5 \\
\hline $\begin{array}{l}\text { Without Pre- } \\
\text { Processing Methods }\end{array}$ & FSVM & 84.1 & 90.7 & 95.8 & 81.3 & 76.7 & 80.0 & 26.7 \\
\hline \multirow{3}{*}{$\begin{array}{l}\text { With Pre-Processing } \\
\text { Method }\end{array}$} & $\begin{array}{l}\text { FSVM-Under } \\
\text { Sampling }\end{array}$ & 74.4 & 87.0 & 88.3 & 64.7 & 66.7 & 71.7 & 33.3 \\
\hline & $\begin{array}{l}\text { FSVM- Over } \\
\text { Sampling }\end{array}$ & 85.6 & 92.6 & 97.9 & 83.3 & 68.3 & 75.0 & 53.3 \\
\hline & $\begin{array}{c}\text { FSVM- } \\
\text { SMOTE }\end{array}$ & 87.8 & 94.4 & 98.3 & 89.3 & 66.7 & 76.7 & 60.0 \\
\hline
\end{tabular}

\section{CONCLUSIONS}

In this paper, a number of intelligent algorithms are developed for determining a health index for the oil-paper insulating system. The health index approach intends to improve the quality of condition assessment of the transformer insulation system by combining results from various chemical and electrical tests, onsite inspections, and information regarding the transformer's operation and loading history. A case study using a real dataset with oil quality tests and DGA test records for 181 in-service power transformers is provided. The case study demonstrates the applicability of the developed intelligent algorithms in determining the health index of the oil-paper insulation system of the in-service transformers. The results illustrate that the FSVM integrated with SMOTE preprocessing method could attain the highest classification accuracy.

\section{REFERENCES}

[1] Z. Xiang and E. Gockenbach, "Asset-Management of Transformers Based on Condition Monitoring and Standard Diagnosis", IEEE Electr. Insul. Mag., Vol. 24, No.4, pp. 26-40, 2008.

[2] V. G. Arakelian, "Effective diagnostics for oil-filled equipment", IEEE Electr. Insul. Mag., Vol. 18, No.6, pp. 26-38, 2002.

[3] M. Fischer, S. Tenbohlen, M. Schafer, and R. Haug, "Determining power transformers' sequence of service in power grids", IEEE Trans. Dielectr. Electr. Insul., Vol. 18, pp. 1789-1798, 2011.

[4] M. Wang, A. J. Vandermaar, and K. D. Srivastava, "Review of condition assessment of power transformers in service", IEEE Electr. Insul. Mag., Vol. 18, No.6, pp. 12-25, 2002.

[5] A. Jahromi, R. Piercy, S. Cress, J. Service, and W. Fan, "An approach to power transformer asset management using health index", IEEE Electr. Insul. Mag., Vol. 25, No.2, pp. 20-34, 2009.

[6] A. D. Ashkezari, T. K. Saha, C. Ekanayake, and M. Hui, "Evaluating the accuracy of different DGA techniques for improving the transformer oil quality interpretation", Australasian Universities Power Engineering Conf. (AUPEC), pp. 1-6, 2011.

[7] B. Pahlavanpour and I. A. Roberts, "Transformer oil condition monitoring", IEE Colloquium Transformer Life Management (Ref. No. 1998/510), pp. 6/1-6/6, 1998.

[8] B. Pahlavanpour, "Characterisation of insulating oils", IEE Colloquium Characterisation of Dielectric Materials: a Review, pp. 8/1-8/5, 1994.

[9] IEC, "Insulating liquids - Determination of acidity by automatic potentiometric titration, IEC 62021-1, 2003.

[10] IEC, "Measurement of relative permittivity, dielectric dissipation factor and d.c. resistivity of insulating liquids", IEC 60247, 2004.

[11] IEC, "Insulating liquids - Determination of the breakdown voltage at power frequency - Test method", IEC 60156, 1995.

[12] P.Wiklund and B.Pahlavanpour, "Properties of Mineral Insulating Oils in Service", J. Iranian Association of Electr. Electron. Eng., Vol. 7, pp.65-73, 2010 .
[13] IEC, "Mineral oil-impregnated electrical equipment in service -Guide to the interpretation of dissolved and free gases analysis", IEC 60599, 2007.

[14] "IEEE Guide for the Interpretation of Gases Generated in Oil-Immersed Transformers", C57.104, 2008.

[15] "IEEE Guide for Acceptance and Maintenance of Insulating Oil in Equipment", IEEE Std C57.106-2006 (Revision of IEEE Std C57.1062002), pp. 1-36, 2006.

[16] IEC, "Supervision and maintenance guide for mineral insulating oils in electrical equipment", IEC 60422, 2005.

[17] IEC, "Mineral oils - Methods for the determination of 2-furfural and related compounds", IEC 61198, 1993.

[18] J. C. Bezdek, Pattern Recognition with Fuzzy Objective Function Algorithms, Kluwer Academic Publishers, 1981.

[19] M. Pal and G. M. Foody, "Feature Selection for Classification of Hyperspectral Data by SVM", IEEE Trans. Geoscience and Remote Sensing, Vol. 48, pp. 2297-2307, 2010.

[20] V. N. Vapnik, The Nature of Statistical Learning Theory: New York:Springer-Verlag, 1995.

[21] L. Chun-Fu and W. Sheng-De, "Fuzzy support vector machines", IEEE Trans. Neural Networks, Vol. 13, pp. 464-471, 2002.

[22] R. Batuwita and V. Palade, "FSVM-CIL: Fuzzy Support Vector Machines for Class Imbalance Learning", IEEE Trans. Fuzzy Systems, Vol. 18, pp. 558-571, 2010.

[23] Z.Zhou and X.Liu, "Training cost-sensitive neural networks with methods addressing the class imbalance problem", IEEE Trans. Knowledge and Data Eng., Vol. 18, pp. 63-77, 2006.

[24] K. B. N. Chawla and P. Kegelmeyer, "SMOTE: Synthetic minority oversampling technique", J. Artif. Intell. Res., Vol. 16, p. 321, 2002.

[25] C.-C.Chang and C.-J.Lin, "LIBSVM: A library for support vector machines", available: http://www.csie.ntu.edu.tw/ cjlin/libsvm, 2013.

\section{ACKNOWLEDGMENT}

The authors gratefully acknowledge Australian Research Council, Powerlink Queensland, Energex, Ergon Energy, and TransGrid for providing supports for this work.

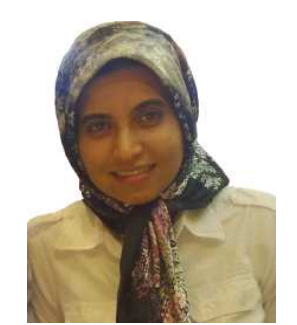

Atefeh Dehghani Ashkezari ( SM'10) was born in Iran. She received the B.Eng. degree (electrical and computer engineering) in 2009 from Sohar University, Oman and the M.Eng. degree (electrical engineering) in 2010 from the University of Queensland, Australia. She completed her master's thesis on non-linearity in transformer diagnosis. She is currently a higher degree student at the University of Queensland. Her research interests include condition monitoring, transformer diagnosis, and power system. 


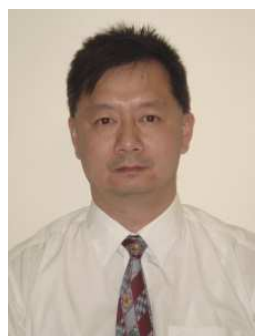

Hui Ma (M'95) received the B.Eng. and M.Eng. degrees from Xi'an Jiaotong University, China in 1991 and 1994, M.Eng. (by research) degree from Nanyang Technological University, Singapore in 1998, and the Ph.D. degree from the University of Adelaide, Adelaide, Australia in 2008. Currently Dr. $\mathrm{Ma}$ is a research fellow in the School of Information Technology and Electrical Engineering, the University of Queensland, Australia. Prior to joining the University of Queensland, Dr. Ma had many years research and development experience. From 1994 to 1995 , he was a researcher in Xi' an Jiaotong University, China. From 1997 to 1999, he worked as a firmware development engineer in CET Technologies Pte. Ltd., Singapore. He was with Singapore Institute of Manufacturing Technology as a research engineer from 1999 to 2003. His research interests include industrial informatics, condition monitoring and diagnosis, power systems, wireless sensor networks, and sensor signal processing.

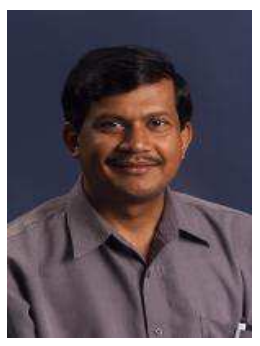

Tapan Kumar Saha (M'93-SM'97) was born in Bangladesh in 1959 and immigrated to Australia in 1989. He received his B.Sc. Eng. degree (electrical and electronic) in 1982 from the Bangladesh University of Engineering \& Technology, Dhaka, Bangladesh, M. Tech (electrical engineering) in1985 from the Indian Institute of Technology, New Delhi, India and the Ph.D. degree in 1994 from the University of Queensland, Brisbane, Australia. Tapan is currently Professor of Electrical Engineering in the School of Information Technology and Electrical Engineering, University of Queensland, Australia. Previously he has had visiting appointments for a semester at both the Royal Institute of Technology (KTH), Stockholm, Sweden and at the University of Newcastle (Australia). He is a Fellow of the Institution of Engineers, Australia. His research interests include condition monitoring of electrical plants, power systems and power quality.

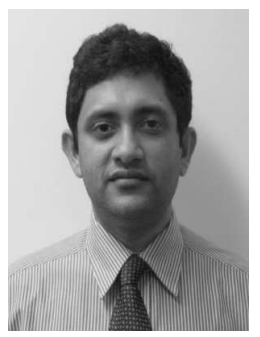

Chandima Ekanayake (M'00) received the B.Sc.Eng.(Hons) degree in 1999 from the University of Peradeniya Sri Lanka. He obtained the Tech. Lic. and Ph.D. degree from Chalmers University of Technology Sweden in 2003 and 2006 respectively. Currently he is a lecturer in the School of Information Technology and Electrical Engineering, the University of Queensland (UQ), Brisbane, Australia. Before joining UQ he was with University of Peradeniya Sri Lanka as a Senior lecturer. During his Ph.D. degree studies he was working for a European Union Project called REDIATOOL where he engaged in research related to Diagnostics of Transformer Insulation from dielectric response measurements. From 2001, he has been involving with condition monitoring of transformers installed at Ceylon Electricity Board, Sri Lanka. He was the Chair of IEEE Sri Lanka Section in year 2006 and 2007. His research interests are condition monitoring of power apparatus, alternatives for insulating oil, transient studies on power systems and energy related studies. 